\title{
A Multiscale Approach to High-Resolution Ocean Profile Observations within a 4DVAR Analysis System
}

\author{
MATTHEW J. CARRIER \\ U.S. Naval Research Laboratory, Stennis Space Center, Mississippi \\ JOHN J. OSBORNE \\ American Society for Engineering Education, Washington, D.C. \\ HANS E. NGODOCK AND SCOTT R. SMITH \\ U.S. Naval Research Laboratory, Stennis Space Center, Mississippi
}

INNOCENT SOUOPGUI

University of New Orleans, New Orleans, Louisiana

JOSEPH M. D’ADDEZIO

Division of Marine Science, School of Ocean Sciences and Technology, University of Southern Mississippi, Stennis Space Center, Mississippi

(Manuscript received 13 October 2017, in final form 21 September 2018)

\begin{abstract}
Most ocean data assimilation systems are tuned to process and assimilate observations to constrain features on the order of the mesoscale and larger. Typically this involves removal of observations or computing averaged observations. This procedure, while necessary, eliminates many observations from the analysis step and can reduce the overall effectiveness of a particular observing platform. Simply including these observations is not an option as doing so can produce an overdetermined, ill-conditioned problem that is more difficult to solve. An approach, presented here, aims to avoid such issues while at the same time increasing the number of observations within the assimilation. A two-step assimilation procedure with the four-dimensional variational data assimilation (4DVAR) system is adopted. The first step attempts to constrain the large-scale features by assimilating a set of super observations with appropriate background error correlation scales and error variances. The second step then attempts to correct smaller-scale features by assimilating the full observation set with shorter background error correlation scales and appropriate error variances; here the background state is taken as the analysis from the first step. Results using a real high-density observation set from underwater gliders in the region southeast of Iceland, collected during the 2017 Nordic Recognized Environmental Picture (NREP) experiment, will be shown using the Navy Coastal Ocean Model 4DVAR (NCOM-4DVAR).
\end{abstract}

\section{Introduction}

The horizontal resolution of modern ocean models has increased significantly over the past decade. Model resolution of $3 \mathrm{~km}$ for global- and basin-scale domains and as high as $1 \mathrm{~km}$ to $500 \mathrm{~m}$ for coastal applications are not uncommon. At the same time ocean observing

Corresponding author: Matthew J. Carrier, matthew.carrier@ nrlssc.navy.mil platforms have begun to provide high-resolution observations of near-surface currents (HF radar; Paduan and Graber 1997), subsurface temperature and salinity profiles (gliders; Rudnick et al. 2004), and, in the near future, sea surface height (SSH) observations from wide-swath altimeters (i.e., Surface Water and Ocean Topography mission; Fu and Ubelmann 2014). However, despite the advancements in modeling capabilities and observations, most operational data assimilation systems remain tuned specifically for constraining mesoscale 
features or larger (Cummings 2005). The inherent problem going forward is, how can the data assimilation and observation processing be altered so that it can continue to constrain mesoscale features while also assimilating high spatial density observations that may contain information on a small-scale phenomenon?

There has been some work within the community to address this so-called multiscale data assimilation problem. One such method, focusing on the threedimensional variational data assimilation (3DVAR) technique, is presented by $\mathrm{Li}$ et al. $(2015 \mathrm{a}, \mathrm{b})$. Li et al. $(2015 \mathrm{a}, \mathrm{b})$ introduce a multistep assimilation approach where the cost function is decomposed into specified large and small scales. The decomposed cost functions are minimized sequentially in order to constrain the large scale using a coarse set of observations, while dense observations are used to constrain small-scale features. This system has been used with the Regional Ocean Modeling System (ROMS; Shchepetkin and McWilliams 2003, 2005; Marchesiello et al. 2001) to support a coastal ocean observing system $(\mathrm{Li}$ et al. 2015a) with good success. This approach was adopted by Miyazawa et al. (2017) for high-resolution satellite sea surface temperature assimilation into an operational ocean forecast system. And Muscarella et al. (2014) extended the method to the Navy Coastal Ocean Model (NCOM; Martin 2000). A similar two-step analysis method was adopted by Xie et al. (2011) for atmospheric forecasting purposes and also by $\mathrm{Xu}$ et al. (2016), where idealized comparisons to one-step analysis methods are performed.

It is generally believed, however, that a more advanced data assimilation method, such as the fourdimensional variational data assimilation (4DVAR) method, would be less affected by the multiscale issue. This is because the 4DVAR is able to resolve fine structures via the dynamic error covariance produced by the action of the tangent linear and adjoint models. However, even the 4DVAR method employs a static error covariance at the initialization time $(\mathrm{Li}$ et al. 2015a); and in the case of the weak-constraint method, throughout the integration of the tangent linear model as well. There are two aspects at play here: the scales represented by the covariance and the processing of observations. It is true that the static covariance in 4DVAR is typically built with one prescribed length scale, however, the action of the tangent linear and adjoint models recover all the scales present in the background model. This effectively makes the dynamic covariance in $4 \mathrm{DVAR}$ multiscale by nature. The issue arises with the processing of observations. Typically, observations are reduced in number when one or more observations are within a length scale distance of one another; with the length scale defined by the static covariance used in the 4DVAR. This is done because dense observations can represent small-scale features that typically have contradictory information (i.e., a temperature innovation that is strongly positive within close proximity to a temperature innovation that is strongly negative). The adjoint handles this well; however, when the adjoint is convolved with the static covariance (with its large prescribed correlation length scale) the result is noisy and unrealistic perturbations to the tangent linear model. This can cause instabilities within the tangent linear model and/or poor convergence.

To counteract this shortcoming in the 4DVAR algorithm, a two-step assimilation process, similar to that in Li et al. (2015a,b), is adopted for the NCOM-4DVAR analysis system. For convenience this implementation will be referred to as multiscale 4DVAR (MS-4DVAR), whereas the standard 4DVAR will be denoted as STD4DVAR. This approach generates increments to the large scale, while simultaneously preserving smaller-scale features in the analysis increments, while also greatly increasing the number of observations that are used in the analysis. This method is tested using the NCOM-4DVAR and a set of high-spatial-density underwater glider observations collected during the Nordic Recognized Environmental Picture (NREP) 2017 experiment. Observations collected from six gliders during a 21-day time period are assimilated using both the STD-4DVAR and the MS-4DVAR assimilation methods. The analyses and resulting 24-h forecasts are compared against the available glider observations.

It should be noted that there is more to the multiscale data assimilation problem than the observation processing and the assimilation procedure itself; however, this is the focus of the present study. How best to define the error correlation length scales of the "large" and "small" scales is important as this will play a large role in the effectiveness of the multistep algorithms presented by Li et al. (2015a,b) and this present study. Also, the dynamical balance relationships change when going from large to small scales; for example, balanced submesoscale currents cannot be sufficiently constrained by the simple geostrophic approximation (Capet et al. 2008). Defining these constraints is important for 3DVAR and can be useful for 4DVAR as well. While this present work introduces a framework within which the multiscale assimilation can be done, solving these other important questions is left for future studies.

In the next section the ocean model, base assimilation system, and observations are introduced. Section three provides a detailed description of the MS-4DVAR assimilation procedure. Section four introduces the experiment setup and provides a detailed description of the results. Section five provides a recap of the problem, 
the findings from the experiment, and a few remarks on future work.

\section{Ocean model, observations, and the standard data assimilation system}

\section{a. Navy Coastal Ocean Model (NCOM)}

NCOM is the operational regional and coastal ocean model for the U.S. Navy. For basin-scale applications NCOM typically runs at roughly $3.5-\mathrm{km}$ resolution; for coastal applications the resolution can be as fine as $500 \mathrm{~m}$. NCOM is a primitive equation model and uses the hydrostatic and Boussinesq approximations. The model has a free surface and is configurable to have terrain-following sigma surfaces overlaid on constant depth-level surfaces in the vertical. This particular model setup utilized the Mellor-Yamada level-2.5 turbulence closure [as described by Kantha and Clayson (2004)] and the Smagorinsky horizontal diffusion schemes (Smagorinsky 1963). Lateral boundary conditions are provided by the Global Ocean Forecasting System (GOFS), which uses HYCOM at $1 / 12^{\circ}$ horizontal resolution and the Navy Coupled Ocean Data Assimilation (NCODA) 3DVAR for data analysis. Surface atmospheric forcing is provided by the Navy's operational global atmospheric model, the Navy Global Environmental Model (NAVGEM; Hogan et al. 2014) at 3-hourly intervals.

The model domain for the experiments presented here covers the North Atlantic between Iceland and the upper United Kingdom, from $55^{\circ}-67.96^{\circ} \mathrm{N}$ to $20^{\circ} \mathrm{W}-$ $3.93^{\circ} \mathrm{E}$ (Fig. 1). The model uses a spherical projection with a horizontal resolution of $3 \mathrm{~km}$ with 50 levels in the vertical (of which half are sigma layers) extending to a maximum depth just past $4000 \mathrm{~m}$. At 3-km resolution, the ocean model is not quite able to resolve submesoscale features. This resolution was selected, however, to ease the computational burden during the testing and evaluation of the MS-4DVAR method. A thorough evaluation of the ability of the MS-4DVAR to properly include more profile observations than the standard configuration is not affected by the choice of resolution, however, since the error correlation length scale is largely responsible for reducing the number of available profile observations to the assimilation.

\section{b. Glider observations}

This model domain covers the region where the NREP experiment is run from 31 May to 24 June 2017. NREP17 deployed, among other devices, a series of six underwater gliders (named Maria, Noa, Dora Jr., India, Rose, and Laura). These gliders were deployed within the region known as the Iceland-Faroe Front (IFF), a

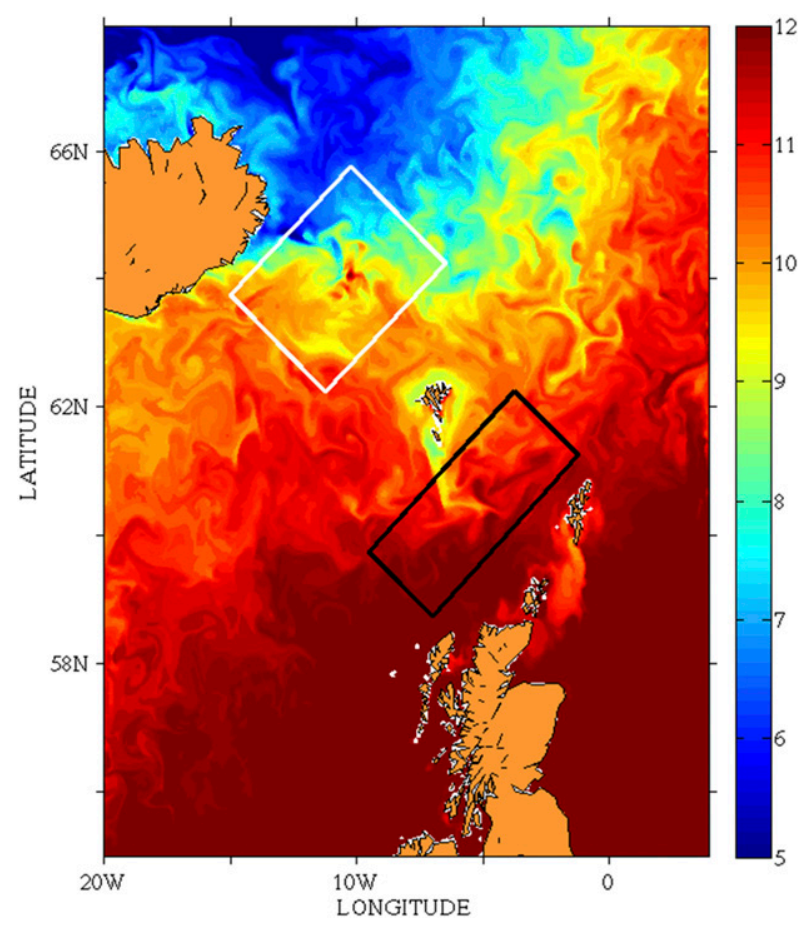

FIG. 1. NCOM domain used for this experiment. Model SST, valid on 21 Jun 2017 (in ${ }^{\circ} \mathrm{C}$, values indicated by color bar), is shown in color contours. Iceland-Faroe Front (IFF) region indicated by white box; Iceland-Shetland Channel (ISC) region indicated by black box.

strong dynamical ocean feature that is formed by the outflow of cold Nordic waters to the north and relatively warm Atlantic water to the south (white box in Fig. 1). It is here that relatively small-scale ocean features $(10-20 \mathrm{~km})$ are generated along the strong temperature gradient defined by the front, as shown by the model sea surface temperature (SST; valid on 21 June 2017) in Fig. 1. This provides a suitable test case for the multiscale 4DVAR methodology since these scales are smaller than the error correlation scale used by the STD-4DVAR. During the early portion of NREP17, from 31 May to 6 June, the glider deployment is mainly focused on the Faroe-Shetland Channel (FSC; black box in Fig. 1); the focus later changed to the IFF region from 8 to 21 June. During NREP17, two of the gliders (Laura and India) collected a relatively small number of profiles, while one glider (Dora Jr.) collected observations far from the IFF and FSC regions. Because of this the main focus of this work will be on the results gathered from 8-21 June within the IFF region using three gliders (Maria, Noa, and Rose).

Figure 2 shows the measurement locations for each of the temperature and salinity profiles collected by the subset of three gliders in the vicinity of the IFF. Figure 2a shows each profile color coded for each individual glider 

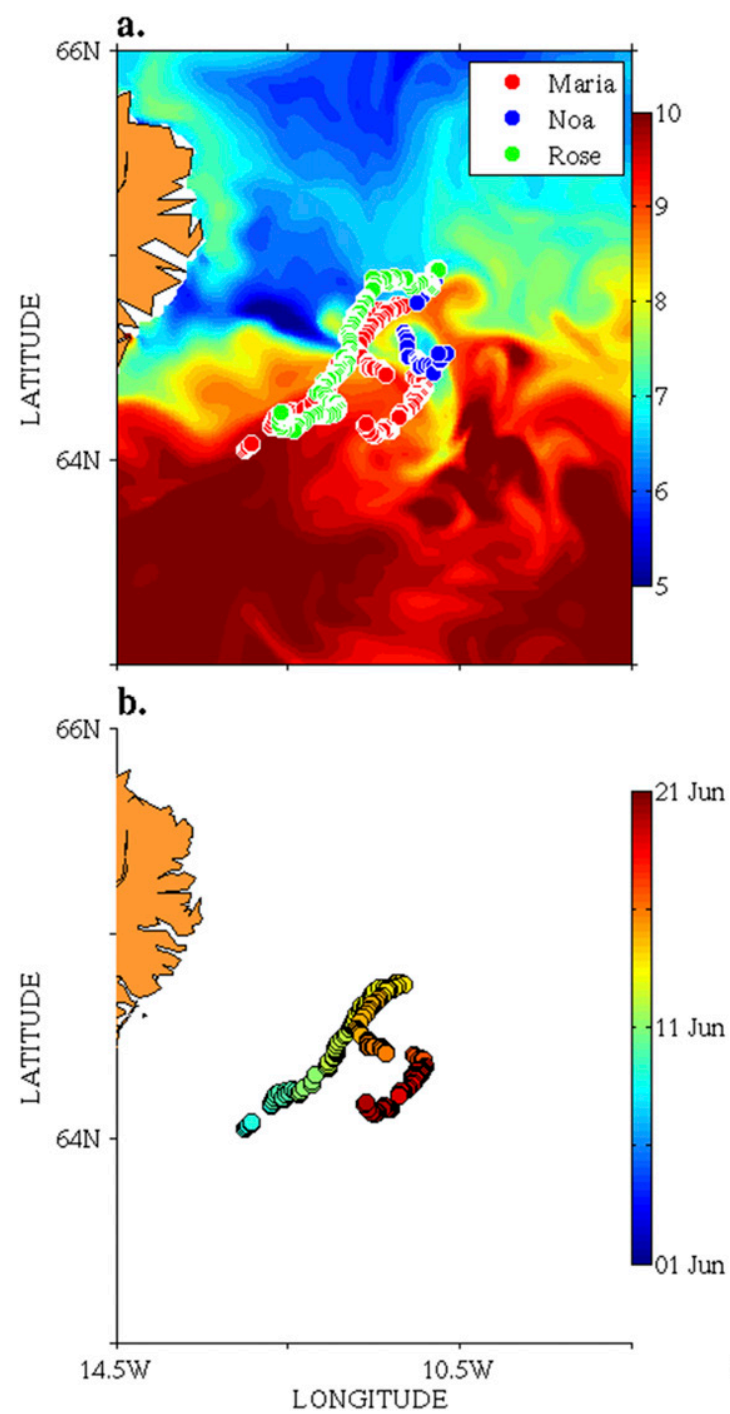

c.

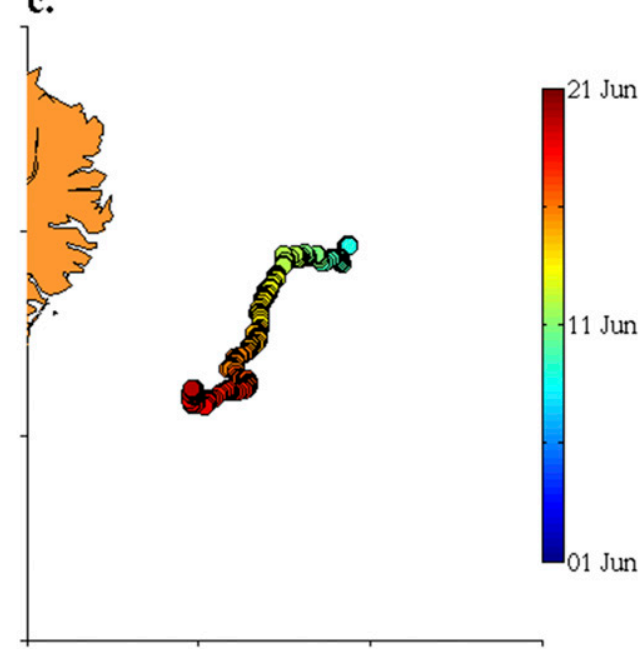

d.

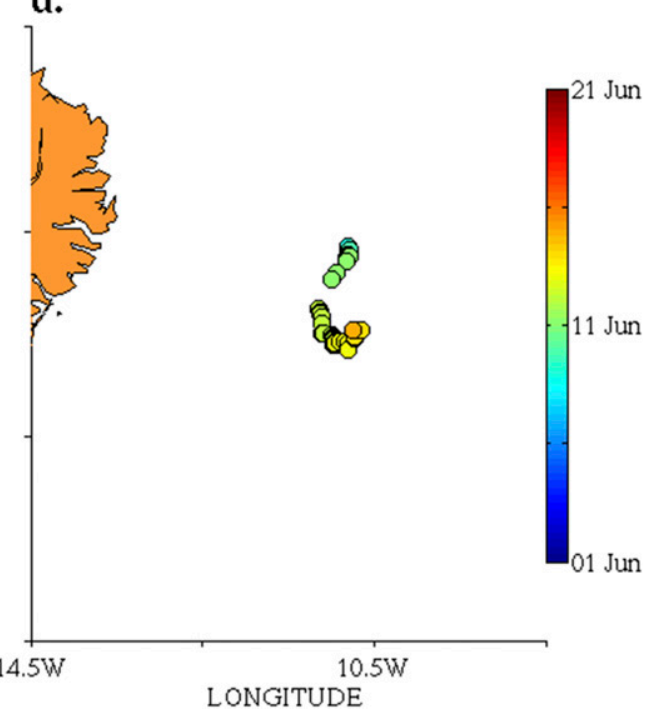

FIG. 2. (a) Location of each glider observation (color coded by the individual glider), overlaid on model SST $\left({ }^{\circ} \mathrm{C}\right.$ ) from 21 Jun 2017. (b)-(d) Individual glider paths within the IFF region (white box in Fig. 1) color coded by the date of the measurement. The gliders that are shown are (b) Maria, (c) Rose, and (d) Noa.

(with the model SST, valid on 21 June 2017, shown in color contours). Figures $2 \mathrm{~b}-\mathrm{d}$ show the path of each individual glider (colored by the date of each measurement). The gliders within this region are Maria (Fig. 2b), Rose (Fig. 2c), and Noa (Fig. 2d); the date of each measurement is indicated by the color bar to the right of each figure. This can be used in conjunction with the temperature and salinity profiles collected from the individual gliders to ascertain when the gliders are crossing the IFF. Figures 3-5 show each temperature and salinity profile from gliders Maria (Fig. 3), Noa (Fig. 4), and Rose (Fig. 5). Glider Maria crosses the IFF on about 12 June, as indicated by its position in Fig. 2 and the transition from warm and salty water to cold and freshwater; it crosses the front again after 18 June. Glider Rose also crosses the IFF, on 15 June, though the transition is from the opposite direction (cold/fresh to warm/salty). Glider Noa's transition is actually due to a change in deployment location from the FSC region to the IFF location. For the comparisons between the STD-4DVAR and MS-4DVAR analyses, the transition across a frontal zone shown in gliders Maria and Rose are particularly useful and will be highlighted in the results discussion in section 4.

\section{c. Standard data assimilation system}

The standard data assimilation system used in this work is the NCOM-4DVAR. NCOM-4DVAR is a weak-constraint analysis system based on the indirect representer method of Bennett $(1992,2002)$ and 

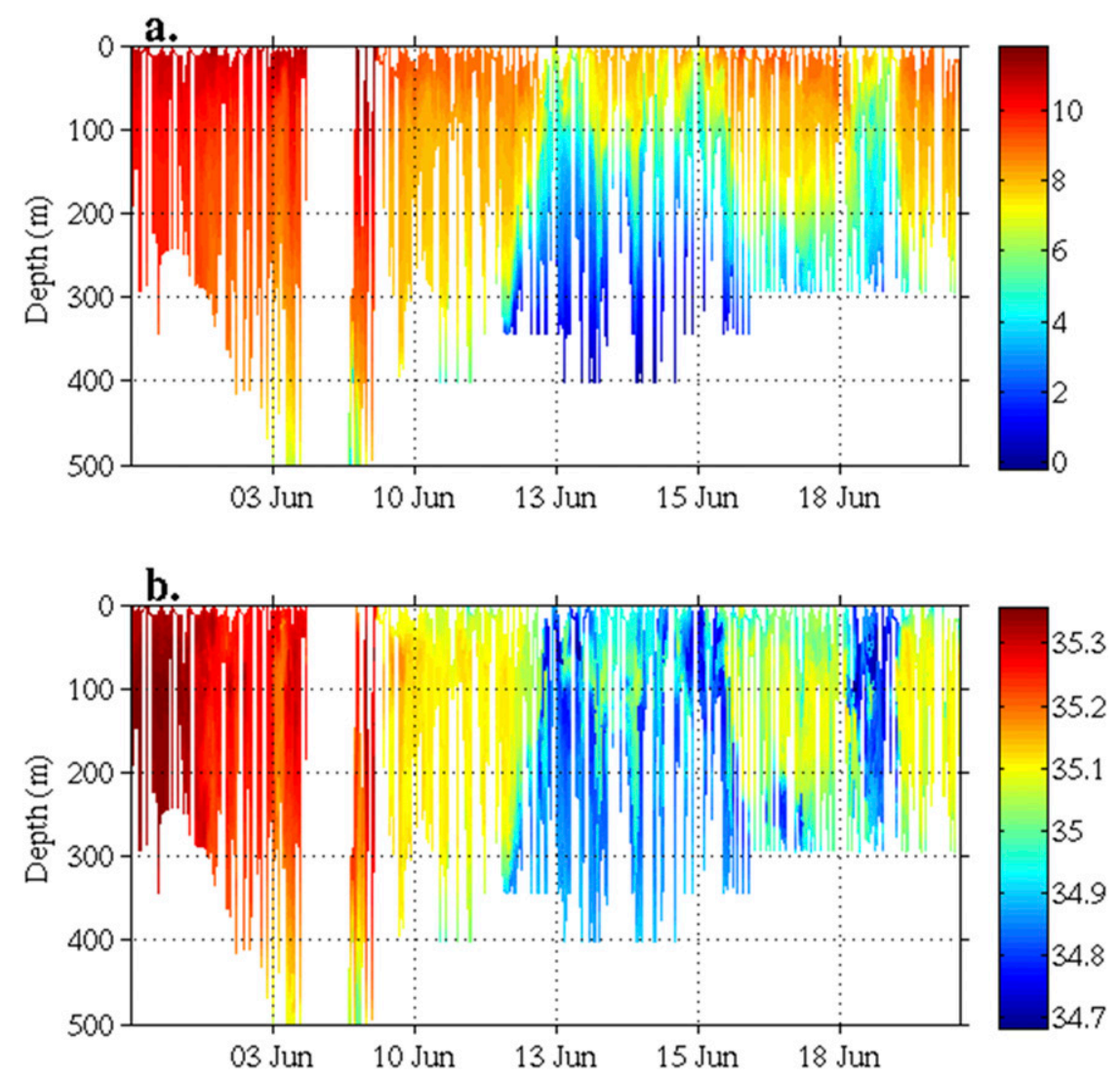

FIG. 3. (a) Temperature and (b) salinity from glider Maria along its travel path during the length of the NREP17 experiment. Values are shown in ${ }^{\circ} \mathrm{C}$ (temperature) and psu (salinity).

Chua and Bennett (2001). The system is described in detail by Ngodock and Carrier (2013, 2014), and Carrier et al. (2014) and a brief description is given here. It is derived from the incremental formulation of the variational cost function (Courtier 1997), time dimension omitted for simplicity:

$$
J(\delta \mathbf{x})=\frac{1}{2} \delta \mathbf{x}^{\mathrm{T}} \mathbf{B}^{-1} \delta \mathbf{x}+\frac{1}{2}(\mathbf{H} \delta \mathbf{x}-\mathbf{d})^{\mathrm{T}} \mathbf{R}^{-1}(\mathbf{H} \delta \mathbf{x}-\mathbf{d}),
$$

where $\delta \mathbf{x}$ is the increment to the state variable, $\mathbf{B}$ is the background error covariance, $\mathbf{H}$ is the observation operator, and $\mathbf{R}$ is the observation error covariance. Here $\mathbf{d}$ is the set of innovations defined as

$$
\mathbf{d}=\mathbf{y}-\mathbf{H} \mathbf{x}^{b},
$$

where $\mathbf{y}$ is the observation set and $\mathbf{x}^{b}$ is the model background. The analysis increment $\delta \mathbf{x}^{a}$ is added to the background $\mathbf{x}^{b}$ to form the analysis solution $\mathbf{x}^{a}$. It can be shown that the so-called dual form of the solution for $\delta \mathbf{x}^{a}$ takes the form of

$$
\delta \mathbf{x}^{a}=\mathbf{B H}^{\mathrm{T}}\left(\mathbf{H B H} \mathbf{H}^{\mathrm{T}}+\mathbf{R}\right)^{-1} \mathbf{d} .
$$

For the indirect representer method, the background error covariance in (3) is expanded to four dimensions (where the vector d now contains observation-model innovations within a time window where $\mathbf{x}^{b}$ and $\delta \mathbf{x}^{a}$ contain the entire model trajectory). This expansion is done by including the action of the adjoint and tangent linear operators, which are derived from the nonlinear model $\mathbf{M}$ such that

$$
\delta \mathbf{x}^{a}=\mathbf{M} \boldsymbol{\Sigma} \mathbf{C} \boldsymbol{\Sigma}^{\mathrm{T}} \mathbf{M}^{\mathrm{T}} \mathbf{H}^{\mathrm{T}}\left(\mathbf{H} \mathbf{M} \boldsymbol{\Sigma} \mathbf{C} \boldsymbol{\Sigma}^{\mathrm{T}} \mathbf{M}^{\mathrm{T}} \mathbf{H}^{\mathrm{T}}+\mathbf{R}\right)^{-1} \mathbf{d},
$$

where $\mathbf{M}$ is the tangent linear model operator, $\mathbf{M}^{\mathrm{T}}$ is the adjoint model, and $\mathbf{\Sigma} \mathbf{C} \boldsymbol{\Sigma}^{\mathrm{T}}$ is a static error correlation $\mathbf{C}$ symmetrically multiplied by the error standard deviation $\Sigma$ that describes the initial condition error or the model error, depending on the portion of the state vector it is applied. This static covariance utilizes an error correlation model based on the implicit solution of a diffusion equation (Weaver and Courtier 2001; Carrier and Ngodock 2010). The NCOM-4DVAR is capable of assimilating temperature, salinity, ocean currents, and sea surface height information from a number of different observing platforms at any frequency 

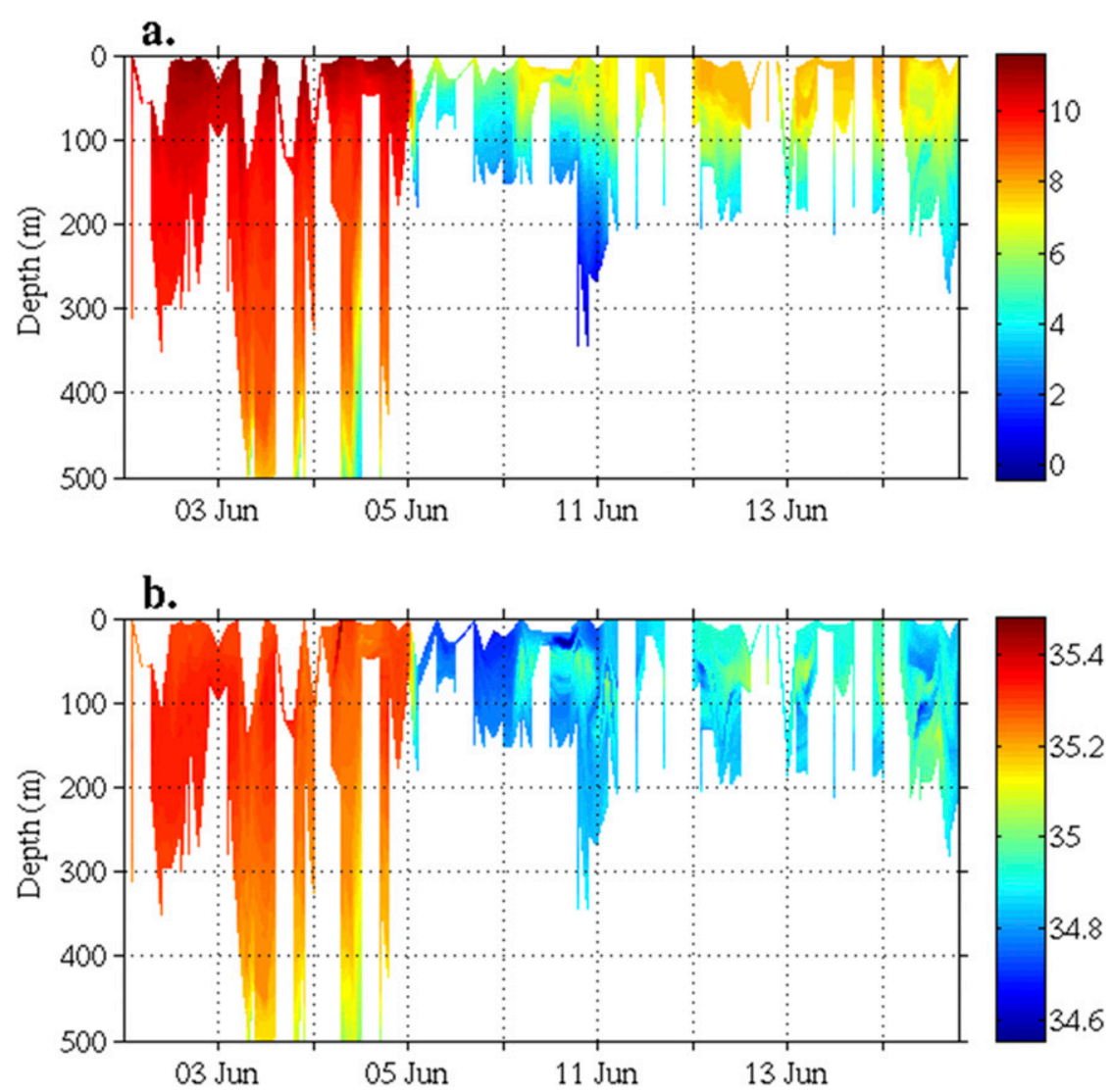

FIG. 4. As in Fig. 3, but for glider Noa.

in time. The system is embedded within the relocatable NCOM system and utilizes the observation preparation software available with NCODA.

\section{Multiscale treatment of observations with NCOM-4DVAR}

STD-4DVAR data processing of spatially dense temperature and salinity profiles proceeds with the assumption that those data that are sufficiently close in time/space are redundant, hence, the application of observation removal within the processing. This observation "thinning" is performed by the NCODA observation preparation software. Ocean temperature profiles, for example, are compared to each other spatially and within a temporal bin (usually 3-hourly bins) by selecting the first profile in the array and determining which other profiles are close to it, as defined by the background error correlation length scale. Those observations that are within this radius are flagged as redundant and only the profile with the deepest and most complete sampling (vertically) is selected; the others are discarded. The algorithm then moves to the next profile in the array (if it had not already been removed) and the procedure is repeated (this time using all the remaining nonflagged profiles). For high-resolution models and observations, however, removal of observations using a single definition of the background error correlation length scale is inappropriate. This is because this processing assumes that the selected profiles are representative of the scales for which the data assimilation is tuned (usually mesoscale or larger). Many times an observation is more representative of some small-scale feature, such as submesoscale eddies or frontal folding. When these type of data are selected for STD-4DVAR, or 3DVAR for that matter, the small-scale information is aliased to the large scale, which degrades the resulting forecast. The opposite can be true, where large-scale information is aliased to the small scale. Both instances are to be avoided in the MS-4DVAR processing and it is largely on these criteria that the algorithm is designed.

MS-4DVAR follows the general two-step assimilation methodology where a set of observations are assimilated in the first step, then the resulting analysis is used as the background for the second step [as in Xie et al. (2011) and $\mathrm{Xu}$ et al. (2016)]. The primary goal here is that the 

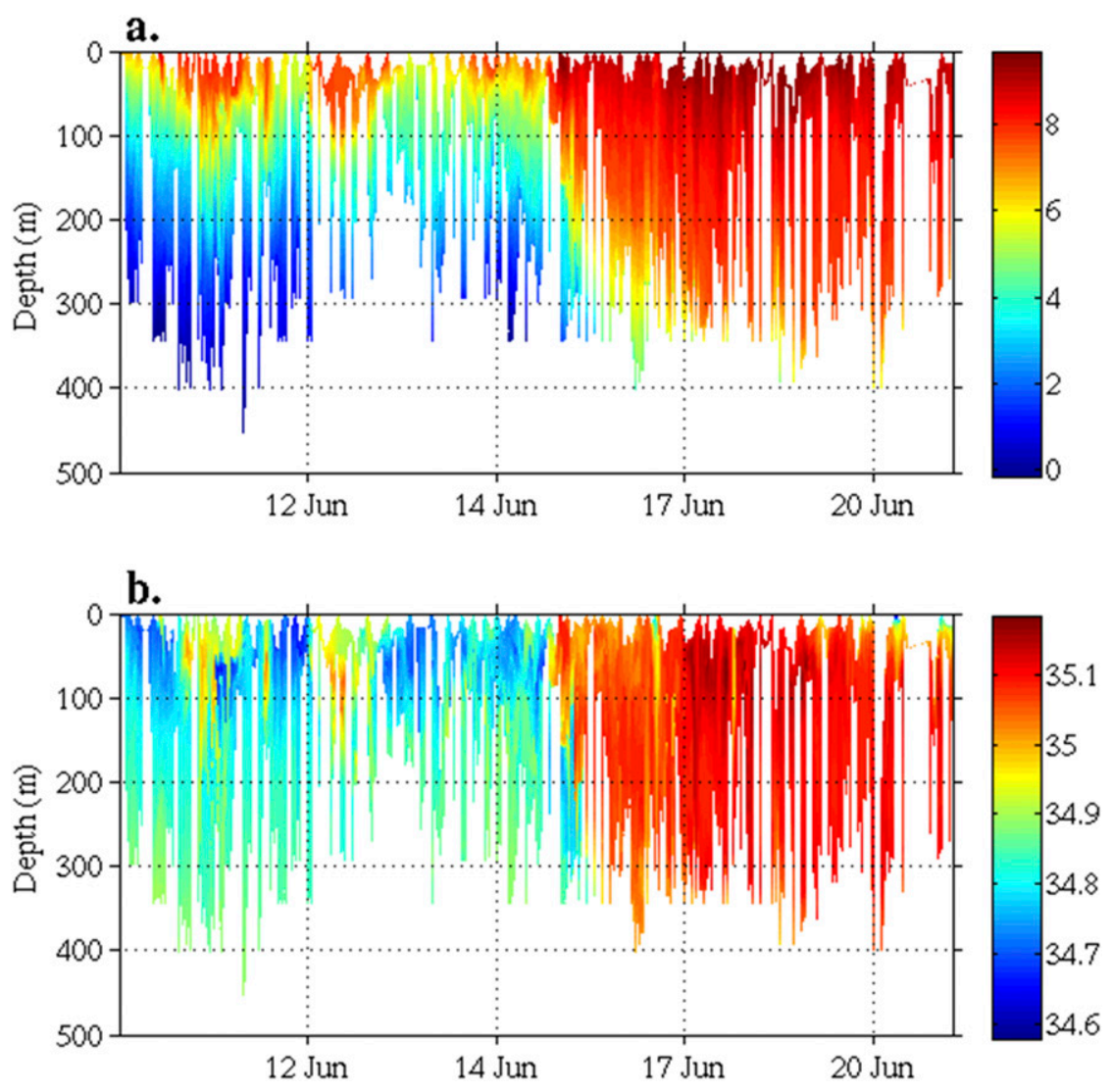

FIG. 5. As in Fig. 3, but for glider Rose.

first step is used to assimilate observations in an attempt to constrain the large-scale features, whereas the second step is used to constrain small-scale features. The MS-4DVAR algorithm is defined by examining the analysis increment generated by a variational data assimilation system,

$$
\delta \mathbf{x}^{a}=\mathbf{K d},
$$

where $\mathbf{d}=\mathbf{y}-\mathbf{H} \mathbf{x}^{b}$ as in (2) and $\mathbf{K}$ is commonly referred to as the Kalman gain matrix, described by the following equation:

$$
\mathbf{K}=\mathbf{B H}^{\mathrm{T}}\left(\mathbf{H B H ^ { \mathrm { T } }}+\mathbf{R}\right)^{-1} .
$$

The covariance B is expanded as in (4) to define the individual tangent linear, adjoint, and correlation operators. The observations can be split into their mean values $\overline{\mathbf{y}}$ and the departures from those mean values $\tilde{\mathbf{y}}$, where the mean value is found by averaging each observation with the surrounding observations that fall within a certain radius defined by the background error correlation scale. If we do this, the innovations can be written as

$$
\overline{\mathbf{y}}+\tilde{\mathbf{y}}-\mathbf{H} \mathbf{x}^{b}=\left(\overline{\mathbf{y}}-\mathbf{H} \mathbf{x}^{b}\right)+\tilde{\mathbf{y}} .
$$

With (7), the "large scale" increment can be defined as

$$
\begin{aligned}
\overline{\delta \mathbf{x}^{a}} & =\mathbf{K} \overline{\mathbf{d}} \\
& =\mathbf{K}\left(\overline{\mathbf{y}}-\mathbf{H} \mathbf{x}^{b}\right) .
\end{aligned}
$$

If (8) is allowed to converge completely, the "small scale" increment can then be found as

$$
\widetilde{\delta \mathbf{x}^{a}}=\mathbf{K}\left[\mathbf{y}-\mathbf{H}\left(\mathbf{x}^{b}+\overline{\delta \mathbf{x}^{a}}\right)\right]
$$

since $\tilde{\mathbf{y}}=\mathbf{y}-\mathbf{H}\left(\mathbf{x}^{b}+\overline{\delta \mathbf{x}^{a}}\right)$ when (8) is fully converged. This derivation makes three key assumptions or approximations. The first assumption is that (8) fully converges; in practice this is not exactly the case. However, if the observation error is sufficiently small and the first assimilation step of the MS-4DVAR is well minimized, this is approximately satisfied (i.e., the analysis residuals are small). The second assumption is that the matrix $\mathbf{K}$ is identical between (8) and (9); in practice they are slightly different. While the operators $\mathbf{M}, \mathbf{M}^{\mathrm{T}}, \mathbf{H}$, and $\mathbf{H}^{\mathrm{T}}$ are identical, $\mathbf{C}$ is different from (8) to (9) as the error 
correlation length scales are shorter in (9); $\boldsymbol{\Sigma}$ and $\boldsymbol{\Sigma}^{\mathrm{T}}$ may also be different (but this is not required). The change made to $\mathbf{C}$ from (8) to (9) is done to ensure that the tangent linear model is not initialized with noisy perturbations, which can arise from convolving an adjoint, forced by spatially dense observations, with a static covariance with a long correlation scale. The third and final assumption is that the mean observations in (7) occupy the same space as $\mathbf{y}$; in practice, the mean observation set $(\overline{\mathbf{y}})$ is smaller than the full set $\mathbf{y}$. The mean observation set is found using an altered version of the NCODA observation thinning algorithm. The alteration made to the algorithm is that instead of removing the redundant observations, they are averaged together to form a profile superobservation (whose location is the average location of the profiles used). The effect of this approximation on the solution is negligible since the full mean observation set contains all of the redundant information; it is this redundancy that is removed in practice. The information that would have been provided by these observations is still carried to $\overline{\delta \mathbf{x}^{a}}$ via the assimilated profile super observations, maintaining the validity of (9). For the results of the experiments to be clear, only the dense glider observations are treated in the multiscale sense; the satellite SST, along-track altimeter SSH, and other profiles are only assimilated within the first step and without averaging. This may result in the analysis drawing too closely to the glider observations in the second step. It can be argued, however, that the background state used in the second assimilation step, which is itself the analysis from the first step and includes the assimilation of satellite SST and SSH observations, acts as a constraint on this portion of the assimilation in order to prevent this; this was not investigated in this study.

The overall cost of the MS-4DVAR algorithm is not prohibitively more expensive than STD-4DVAR; indeed, it is roughly equivalent to the STD-4DVAR when that algorithm employs two outer loops (which is common). Because of the difference in the size of the observation vector between the two assimilation steps, the MS-4DVAR is roughly 1.5-2 times the computational cost of one outer loop of STD-4DVAR. On the Cray XC40 machines where these experiments were run (using 96 processors) the MS-4DVAR averaged $42 \mathrm{~min}$ for each 24 -h analysis-forecast cycle.

The static error covariance for the first assimilation step is set with larger correlation scales (based on the Rossby radius of deformation and scaled up to $25-30 \mathrm{~km})$ and error standard deviation values are based on innovations, calculated using the model and mean observations, in an attempt to capture the large-scale error. This is calculated as a RMS error profile:

$$
\mathrm{RMSE}_{k}=\sqrt{\frac{\sum_{n=1}^{N}\left(\bar{y}_{n}-H_{n} x\right)^{2}}{N}},
$$

where $k$ is the bin depth layer, $N$ is the total number of observations that fall within that bin depth layer, $H_{n}$ is the operator that maps the model solution to the observation location, and $\bar{y}_{n}$ is the vector of mean profile observations that fall with the $k$ th bin. This error is constant horizontally at each layer (with a maximum of $\sim 1.5^{\circ}$ for temperature and $\sim 0.5$ psu for salinity). For the second step in MS-4DVAR the error correlation scales have been set to half that of the first assimilation step. The error values have been computed using the same formula as the first step, but using $y_{n}$ in place of $\bar{y}_{n}$. This produces maximum error values of $\sim 1.75^{\circ} \mathrm{C}$ for temperature and $\sim 0.5 \mathrm{psu}$ for salinity. Observation error is included in both assimilation steps of MS-4DVAR. Typically, NCODA adds a representation error to the prescribed instrument error when processing profile observations. This representation error is included in this study and it attempts to account for the layer averaging that is done to high vertical-resolution profile observations within NCODA. The representation error does not account for small-scale (large-scale) error in the large-scale (small-scale) portion of the two-step analysis (Li et al. 2015b) and this may result in observation overfitting. MS-4DVAR attempts to account for this by how it treats the glider data in the two-step analysis. Those individual gliders that are used to calculate the superobservation in the first step, and are similar to the superobservation itself, contribute mostly to the large-scale analysis. In the second step, their innovations are small as the background state is the analysis from the first step. Similarly, those gliders that are unlike the superobservation have large innovations in the small-scale analysis and contribute mainly to that step. When both analysis steps are complete, the increments from both the large-scale and small-scale analysis are added to the full background to produce the complete analysis solution.

Figure 6 shows the results of assimilating a subset of profile observations from the NREP17 glider dataset just to the east of Iceland. Using a 48-h assimilation window the STD-4DVAR and MS-4DVAR produce analyses that end at 0000 UTC 15 June 2017. Figure 6a shows the temperature analysis increment from STD-4DVAR (color contours) and the temperature innovation (colored circle), from which the increment is generated at $100-\mathrm{m}$ depth at the end of the analysis. 

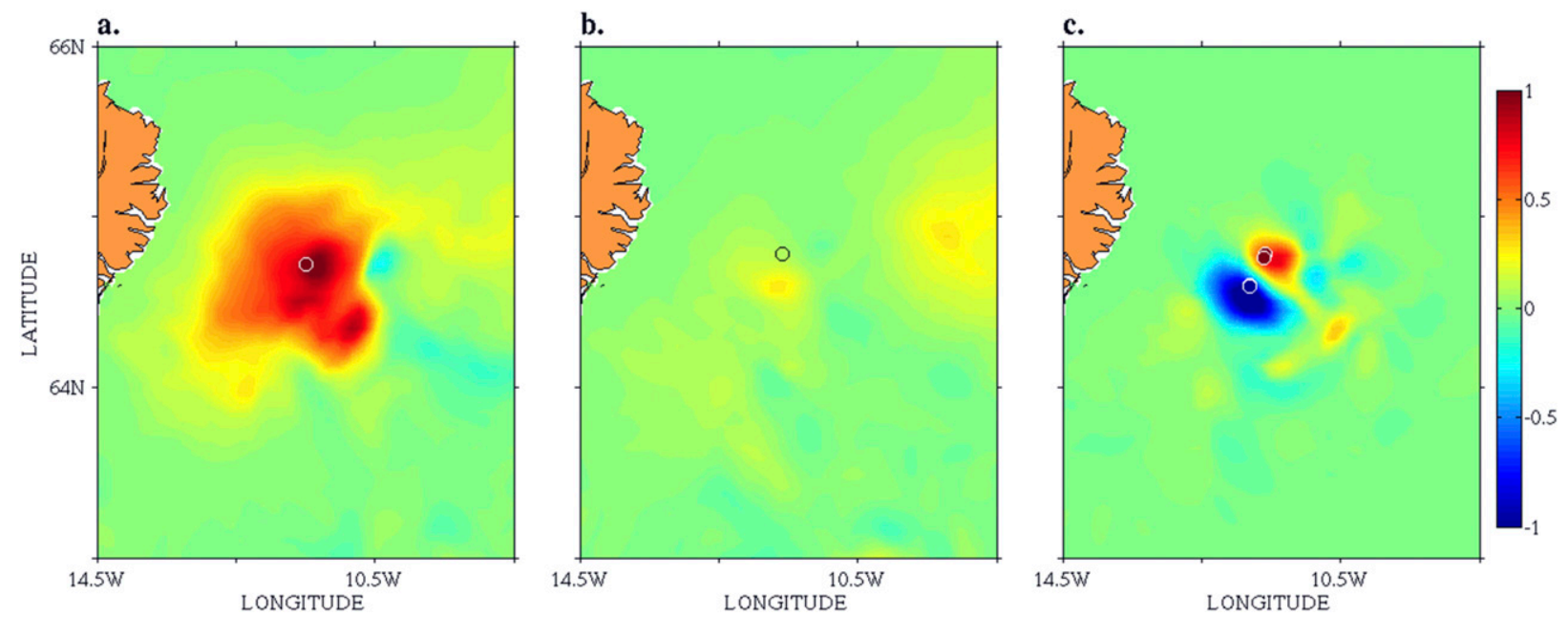

FIG. 6. (a) Temperature innovation (small colored circle) and increment (color-contoured region) from (a) STD-4DVAR, (b) first assimilation step of MS-4DVAR, and (c) second assimilation step of MS-4DVAR at 100-m depth to the east of Iceland at 0000 UTC 15 Jun 2017. Values shown are in ${ }^{\circ} \mathrm{C}$.

At this time, there are numerous glider profile observations available within the assimilation window; however, because of the NCODA observation thinning algorithm, only one profile is used by STD-4DVAR. Clearly the large-scale correlation operator plays a substantial role here as the increment has relatively wide spatial coverage. Figure $6 \mathrm{~b}$ shows the temperature innovation and increment, this time from the first assimilation step of the MS-4DVAR system. Like STD-4DVAR, the first assimilation step of MS-4DVAR has only one profile observation; however, unlike STD-4DVAR, this profile observation is a superobservation formed from all the available profiles in the immediate area. In Fig. 6b, the mean temperature across a local front is close to the value of the model at that location, creating a small innovation that contributes little to this portion of the MS-4DVAR analysis. Figure $6 \mathrm{c}$ shows the increment from the second assimilation step of the MS-4DVAR system using the innovations shown. In this case, the profile observations are not averaged together and are assimilated as separate profiles. It should be noted that the number of profiles may still be reduced if two or more of these profiles fall within a correlation scale length of each other in the second assimilation step. In this case, the profiles would be reduced in number as in STD4DVAR; however, the second step of the MS-4DVAR uses much shorter correlation length scales and as such fewer profiles are considered redundant (more profiles are retained). These data have a substantial impact on the analysis and indicate a small-scale feature with a negative increment to temperature on the west side and a positive increment to the east. Not coincidentally, these observations are taken right along the IFF where cold Nordic water from the north meets warm Gulf Stream water from the south. This information is completely lost in STD-4DVAR, as that system aliases localized information to the large-scale increment. In MS-4DVAR, on the other hand, the increment more accurately reflects the position and magnitude of the front.

\section{Experiment design and results}

\section{a. Experiment design}

The MS-4DVAR and STD-4DVAR analysis implementations are compared to each other using the aforementioned NREP17 glider dataset. The experiments are run from 1 to 21 June 2017 using the glider data in combination with other available satellite SST and SSH observations. These SST and SSH data are assimilated only in the first assimilation step of MS-4DVAR. The assimilation window is $48 \mathrm{~h}$ and the update cycle is repeated every $24 \mathrm{~h}$. The assimilation window length of $48 \mathrm{~h}$ is selected due to tangent linear model stability limitations for this domain. Observations that fall within the first $24 \mathrm{~h}$ of the 48 -h assimilation window are reassimilated (i.e., same observations that were assimilated in the last $24 \mathrm{~h}$ of the previous analysis cycle). It is arguable that this may allow for observation overfitting. However, this is mitigated by the fact that the analysis of the previous cycle is used to calculate the innovations within the overlap. Therefore, the innovations that are calculated within the overlap are the residuals of the previous analysis, are small in magnitude (cf. the innovations from new observations), and should contribute little to the overall cost function. 


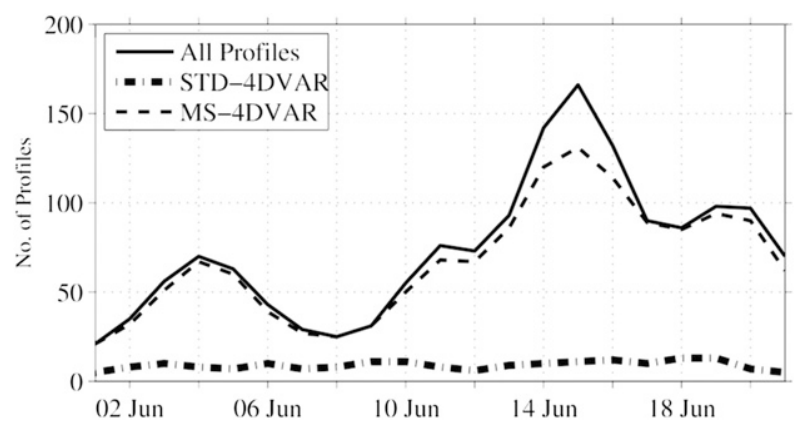

FIG. 7. Glider profile count during each analysis cycle. All available profiles are shown by thick black line, those selected for assimilation in STD-4DVAR are shown in dash-dot black, and those selected for assimilation by in MS-4DVAR (within the second assimilation step) are shown in dashed black.

For STD-4DVAR the observations are processed through the Navy's operational NCODA data processing suite. The static error correlation scales and error variance values in STD-4DVAR are identical to those of the first assimilation step of MS-4DVAR. This results in a drastic difference in the amount of glider observations that make their way into the respective analyses. Figure 7 shows the number of glider profiles that are used during each of the 21 analysis cycles for the STD-4DVAR (dash-dot black line), the second step of MS-4DVAR (black dashed line), and the total number of profiles available (solid black). The number of profiles at each analysis time is very small in STD-4DVAR when compared to the second step of MS-4DVAR, sometimes by an order of magnitude. For the second assimilation step of MS-4DVAR, the number of profiles used in each analysis is very close to the total number, but not exact as some observations are still removed in the second assimilation step if multiple profiles fall within one correlation scale of each other. Overall, the STD-4DVAR uses about $12 \%$ of the total available profiles; the MS-4DVAR uses nearly $91 \%$ of the total available profiles.

\section{b. Experiment results}

The along-glider track temperature and salinity profile figures shown earlier (Figs. 3-5) highlighted some of the small-scale features that exist in the observations (i.e., the temperature gradient across the IFF). It is helpful to look at the analyses from the STD-4DVAR and MS-4DVAR experiments in the same manner. Figures 8 and 9 show the absolute difference between the glider observations and the STD-4DVAR (top panels) and MS-4DVAR (bottom panels) for temperature (left panels) and salinity (right panels) for gliders Maria (Fig. 8) and Rose (Fig. 9). It should be noted that most of these profiles are assimilated in the analysis for MS-4DVAR and not for STD-4DVAR. With that in mind, this comparison is still useful for two reasons. First, it has been the assumption that STD-4DVAR removes much of these data because they are considered redundant, and it has been postulated in this work that this is not always the case. Comparing these two analyses in this manner will determine if this is true; that is, if the
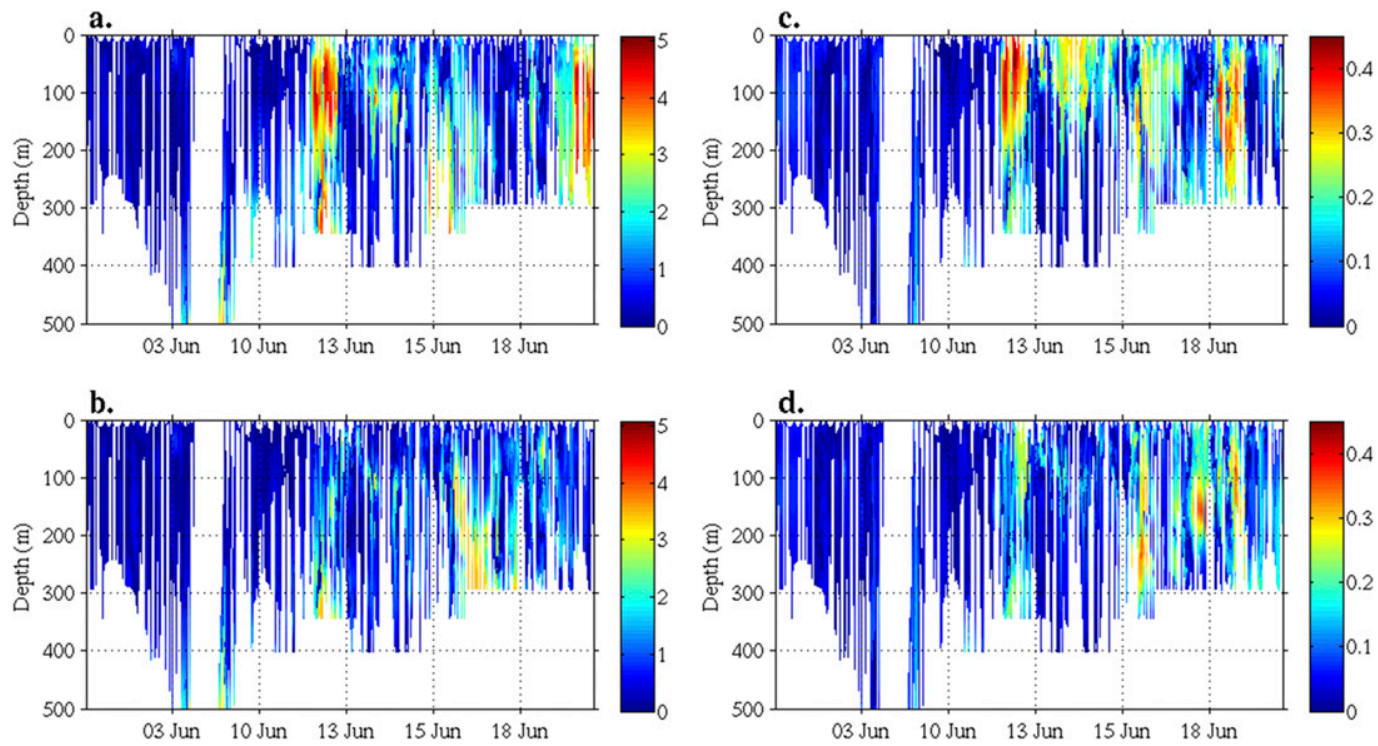

FIG. 8. Absolute difference between model analysis and glider observation (at the observation locations) using glider Maria for (a) STD-4DVAR temperature, (b) MS-4DVAR temperature, (c) STD-4DVAR salinity, and (d) MS-4DVAR salinity. Values are shown in ${ }^{\circ} \mathrm{C}$ (temperature) and psu (salinity). 

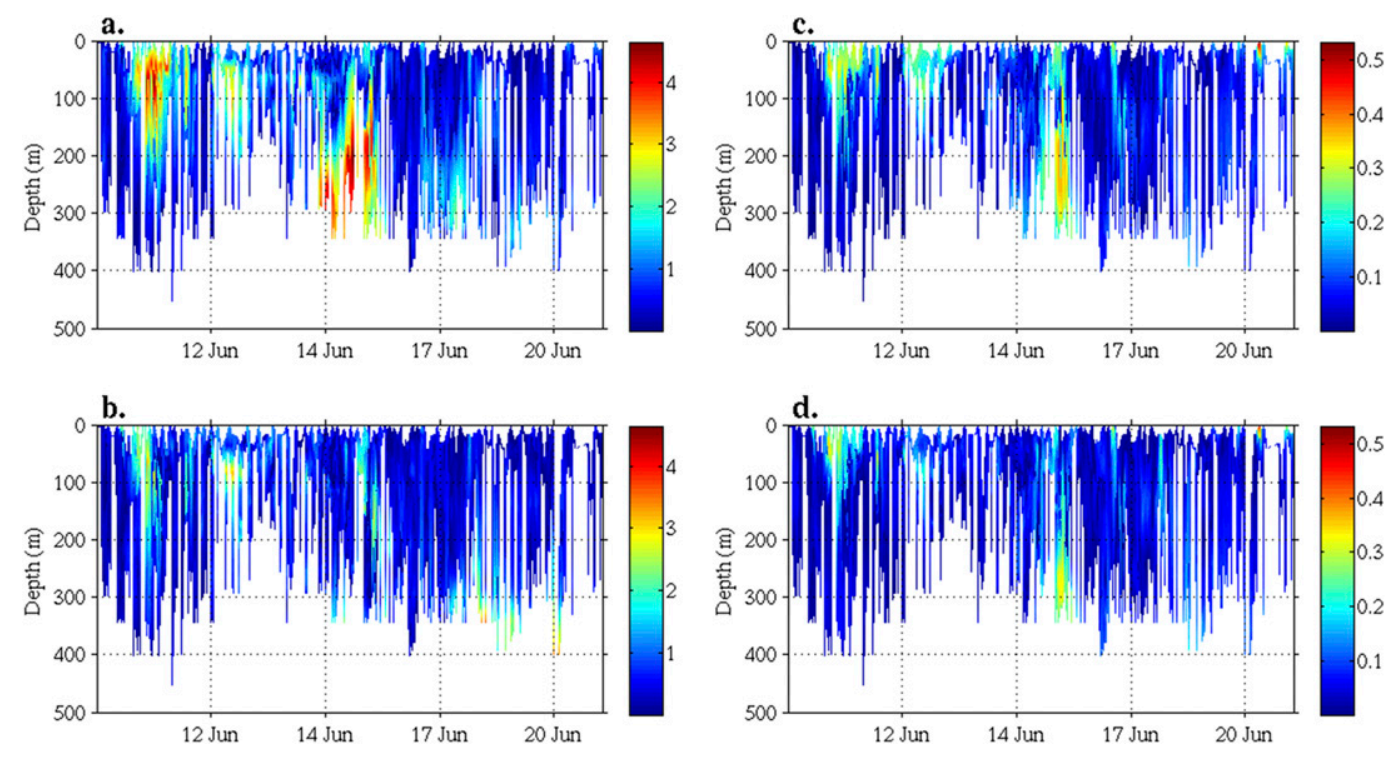

FIG. 9. As in Fig. 8, but for glider Rose.

analyses compare to the observations in the same manner, then these data are, in fact, redundant and a multiscale treatment is not needed. Further, examining the analysis-observation differences along the glider tracks highlight how well the MS-4DVAR is fitting the observations using such a spatially dense dataset. This comparison will clearly expose any erroneous or nonphysical increments generated by MS-4DVAR in the vicinity of the IFF. Examining Figs. $8 \mathrm{a}$ and $8 \mathrm{~b}$, the two analyses compare generally well for much of the glider observations, and each analysis compares well to the glider temperature data itself with most of the differences at or under $1^{\circ} \mathrm{C}$. However, in the vicinity of the IFF (the temperature gradient seen in Fig. 3 around 12 June), there is a marked difference between the two analyses. The STD-4DVAR shows a substantial departure from the glider temperature observations, with difference values as high as $5^{\circ} \mathrm{C}$. This is due to the effect of the large correlation scale used in the static covariance (demonstrated in Fig. 6), which in turn prevents these observations from being included in the assimilation. The MS-4DVAR, on the other hand, does not indicate such a departure with maximum difference with the glider temperature peaking at about $2^{\circ} \mathrm{C}$. This indicates that this information, provided by the glider, is not redundant and can be captured accurately by the MS-4DVAR approach. Much the same is seen in the glider salinity differences (Figs. 8c and 8d) as well.

The same type of behavior is shown when examining the along-glider track difference with Rose. Figure 5 indicates that Rose crosses the IFF around 15 June going from Nordic to Atlantic waters. The STD-4DVAR analysis does not capture this transition well, showing temperature differences with Rose (Fig. 9a) above $4^{\circ} \mathrm{C}$, mostly in the deeper portion of the ocean around $150-300-\mathrm{m}$ depth. This would suggest that the STD-4DVAR analysis is not capturing the slope of the IFF at depth. The MS-4DVAR captures this better with temperature differences (Fig. 9b) closer to $1^{\circ}-2^{\circ} \mathrm{C}$ (and again, Figs. $9 \mathrm{c}$ and $9 \mathrm{~d}$, indicate similar behavior in salinity). The comparisons to Maria and Rose show that the STD-4DVAR and MS-4DVAR do a very good job of fitting the glider observations in regions away from the IFF transition (as indicated by the very low differences between the analyses and glider observations in these regions). This suggests that features in these areas are well represented by large-scale error covariance assumptions. However, along the IFF transition, only MS-4DVAR does well in capturing the temperature and salinity structure and magnitudes.

To illustrate the scale of the frontal structures captured by the MS-4DVAR, the analysis for both STD-4DVAR and MS-4DVAR on 13 June 2017 for glider Maria is highlighted. This is just after glider Maria moved across the IFF from Atlantic waters to colder Nordic waters (cf. Fig. 8). Figure 10 shows the analysis temperature from STD-4DVAR at 100-m depth at 0000 UTC 13 June 2017 (Fig. 10a), MS-4DVAR (Fig. 10c), the STD4DVAR temperature increment at the end of the analysis cycle that ended at 0000 UTC 13 June (Fig. 10b), and the corresponding MS-4DVAR increment (Fig. 10d). Figures 10a and 10c both show the glider observations overlaid in colored circles (observations from the time period of 11-13 June 2017); Fig. 10b has 

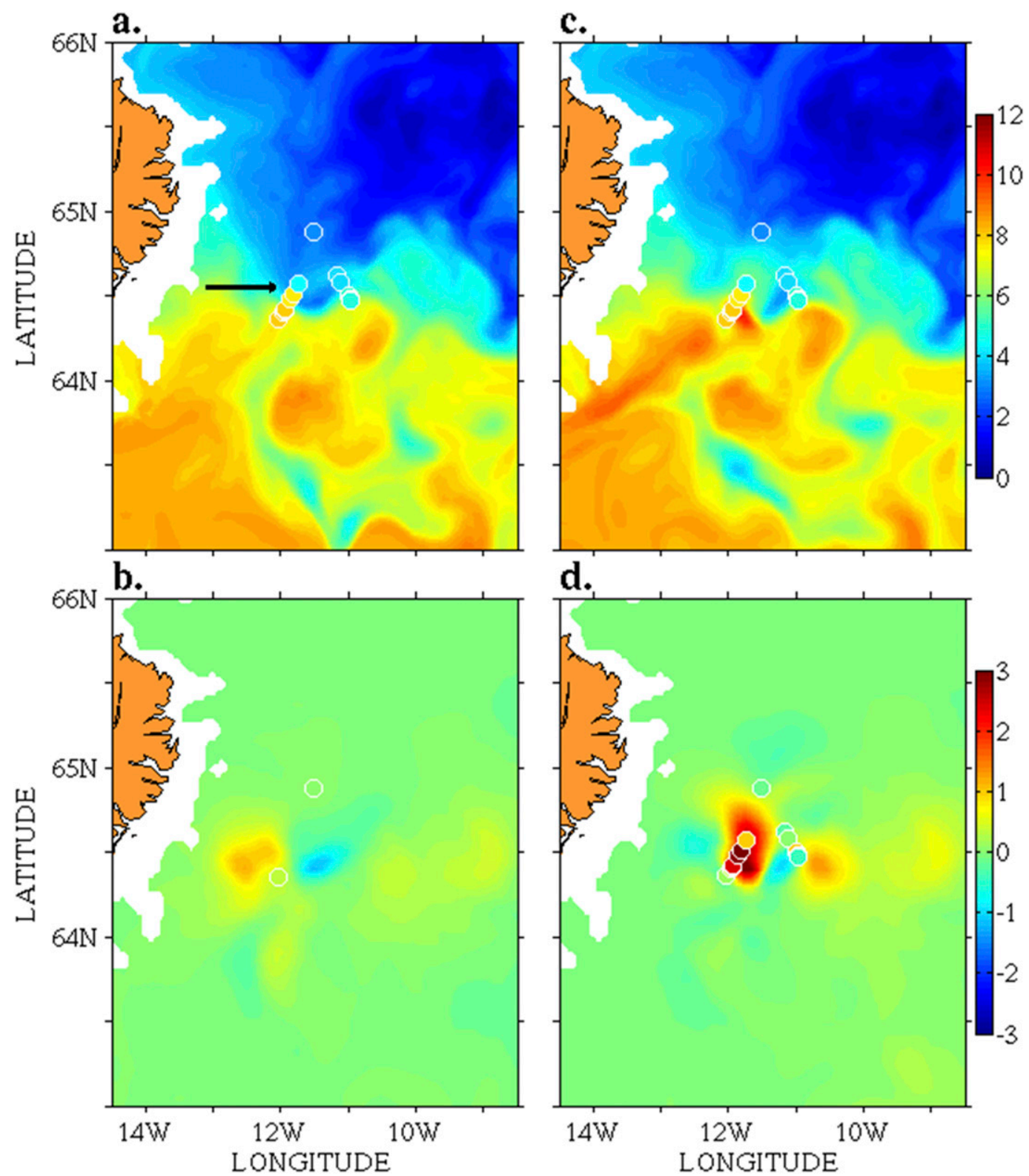

FIG. 10. Model analysis fields at 100-m depth from 0000 UTC 13 Jun 2017 to the east of Iceland. (a) Temperature from STD-4DVAR (with glider observations valid at this time shown in colored circles), (c) temperature from MS-4DVAR, (b) corresponding temperature increment field from STD-4DVAR (with assimilated glider innovations shown in colored circles), and (d) temperature increment field from MS-4DVAR (with assimilated glider innovations shown in colored circles). Values are shown in ${ }^{\circ} \mathrm{C}$.

the innovations (colored circles) used during the 13 June analysis (innovations from the time period of 11-13 June analysis window) for STD-4DVAR; and Fig. 10d shows the same as Fig. 10b, but for MS-4DVAR. Figure 10a shows (black arrow) that the IFF is too far south in the vicinity of the glider observations in STD-4DVAR (cold water indicated by the analysis, gliders show relatively warmer water). This is not the case with MS-4DVAR, as there is a warm tongue protruding northward in the vicinity of the glider observations. This is explained by examining the observations that were used in the analysis of both systems. Figure $10 \mathrm{~b}$ shows that STD4DVAR only uses observations that are well north and south of the Nordic-Atlantic water interface, with the innovations indicating that the background state already fits these observations well. MS-4DVAR (Fig. 10d), on the other hand, is able to utilize all of the glider observations that cross the IFF. In doing so, 

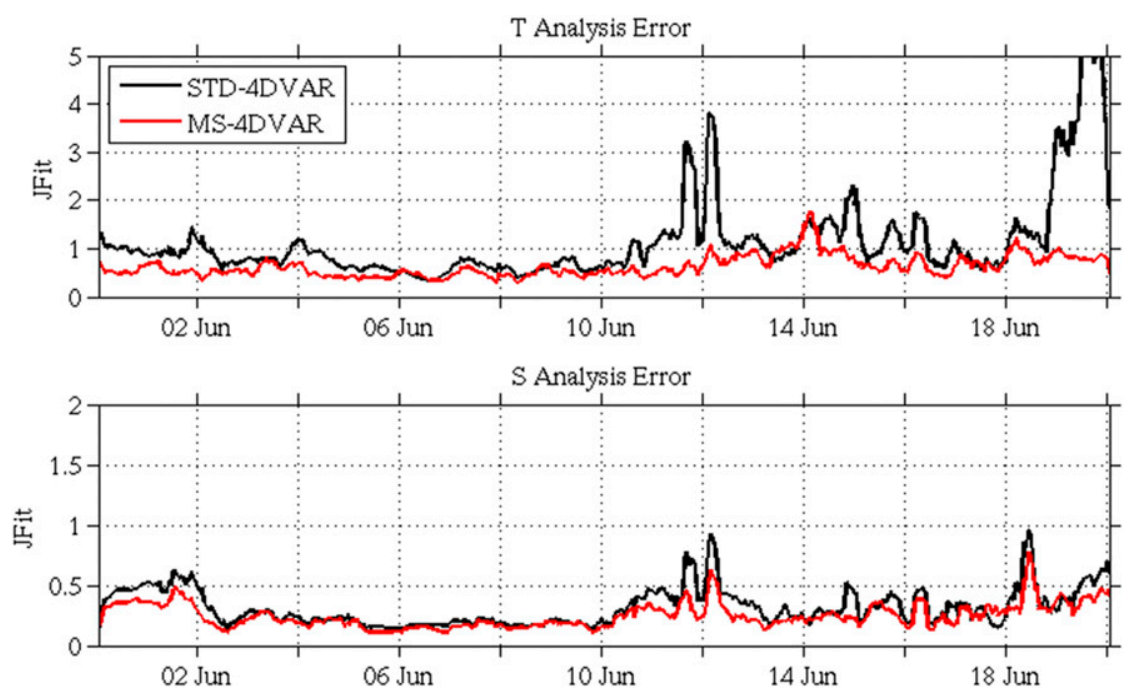

FIG. 11. $J_{\text {FIT }}$ metric [see Eq. (6)] for STD-4DVAR (black lines) and MS-4DVAR (red lines) for (top) temperature and (bottom) salinity throughout the experiment time frame (1-21 Jun 2017). Values are computed by interpolating model analysis fields to glider observation locations. Gliders used in MS-4DVAR assimilation are shown here.

the MS-4DVAR analysis is able to correct the model background state and produce a temperature increment that matches the data more closely.

The relative performance of each analysis can be examined statistically by comparing the analyses from STD-4DVAR and MS-4DVAR to each glider observation throughout the experiment using the following $J_{\text {FIT }}$ metric (Ngodock and Carrier 2014):

$$
J_{\mathrm{FIT}}=\frac{1}{M} \sum_{m=1}^{M} \frac{\left|\mathbf{y}_{m}-\mathbf{H}_{m} \mathbf{x}^{a}\right|}{\boldsymbol{\sigma}_{m}},
$$

where $\mathbf{y}_{m}$ is the $m$ th glider observation, $\mathbf{H}_{m}$ is the observation operator, $\mathbf{x}^{a}$ is the analysis, $\boldsymbol{\sigma}_{m}$ is the observation standard deviation error, and $M$ is the total number of observations. The $J_{\mathrm{FIT}}$ metric is based on the notion that a data assimilation system should fit the assimilated observations within the assumed observation standard deviation. If $J_{\mathrm{FIT}}$ is at or below 1.0 then the resulting analysis has satisfied this criterion. Figure 11 shows the $J_{\text {FIT }}$ metric for the STD-4DVAR (black line) and MS-4DVAR (red line) for temperature (top panel) and salinity (bottom panel) as compared to all glider observations that were included in the MS-4DVAR analysis from 1 to 21 June 2017. The $J_{\text {FIT }}$ metric is computed every $3 \mathrm{~h}$ (all observations that fall $\pm 1.5 \mathrm{~h}$ around each bin time are used and define $M$ ). Again, it is noted that STD-4DVAR has not assimilated all of these glider observations, therefore, it is not expected that STD-4DVAR will have a $J_{\text {FIT }}$ value at or below 1.0; however, as stated previously, this is a good indication of how redundant the glider observations are: if the profile observations that are removed in STD-4DVAR are truly redundant with those that are retained, the $J_{\mathrm{FIT}}$ values of STD-4DVAR should be similar to that of MS-4DVAR. For salinity, both STD-4DVAR and MS-4DVAR do a good job overall with $J_{\text {FIT }}$ values below 1.0 for both analyses throughout the experiment time frame. For temperature, however, STD-4DVAR exhibits high $J_{\text {FIT }}$ values past 10 June (when the gliders are concentrated within the IFF region). Generally speaking, the MS-4DVAR analysis fits all the observations within the observation error, indicating again that this method does better in capturing the small-scale features represented in the data. A more fair comparison would be to examine the analysis fit to observations that are independent from both analyses. Figure 12 shows this, as nearly $10 \%$ of the total number of glider profiles is not included in either analysis. Here the mean absolute error (MAE) is computed, again every $3 \mathrm{~h}$ using observations at $\pm 1.5 \mathrm{~h}$ around each bin time. The error values are very similar between MS-4DVAR and STD-4DVAR for the time period prior to 10 June, before the gliders enter the IFF region. Once the gliders begin sampling the IFF region, however, the MS-4DVAR analysis exhibits generally lower error than STD-4DVAR for both temperature and salinity, with temperature error never exceeding $1^{\circ} \mathrm{C}$, whereas STD-4DVAR has errors as high as $1.5^{\circ} \mathrm{C}$.

It is important to see if the gains in the assimilation for MS-4DVAR have any appreciable difference on the resulting forecast. It should be noted, however, that a 

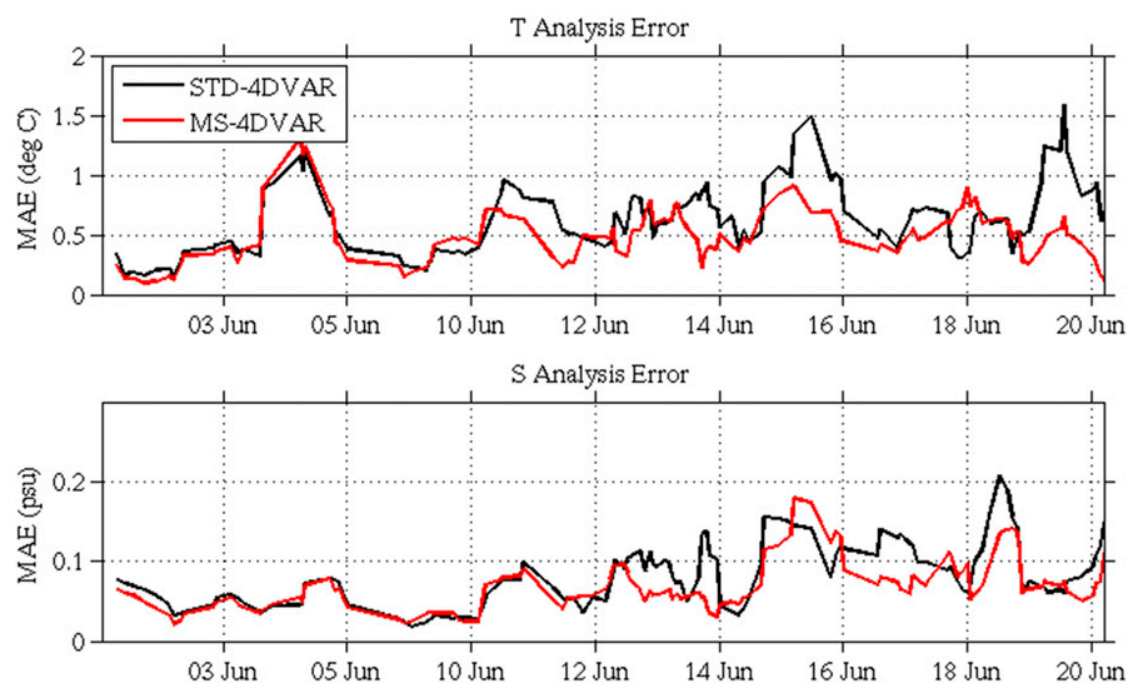

FIG. 12. Mean absolute error (MAE) for STD-4DVAR (black lines) and MS-4DVAR (red lines) for (top) temperature and (bottom) salinity throughout the experiment time frame (1-21 Jun 2017). Values are computed by interpolating model analysis fields to glider observation locations. Gliders not used in either experiment (i.e., independent observations) are shown here.

superior forecast is desirable when updating a data assimilation scheme, but not always possible. This is especially true for regional models, such as NCOM, where lateral and surface boundary conditions also play an important role along with the initial condition. In this present study, forecasts from MS-4DVAR should reasonably outperform those from STD-4DVAR in regions where the feature scales are relatively small (i.e., in the vicinity of the IFF) and where there are observations to sample these features. Away from these locations, the forecast from MS-4DVAR should be very similar to that from STD-4DVAR. To examine this the 24-h forecast error is computed for forecasts generated from both the STD-4DVAR and MS-4DVAR analyses. The MAE is used again and this time the metric is computed every $24 \mathrm{~h}$ (using those observations that fall at $\pm 12 \mathrm{~h}$ around each bin time). Figure 13 shows this for STD-4DVAR (black line) and MS-4DVAR (red line) for temperature
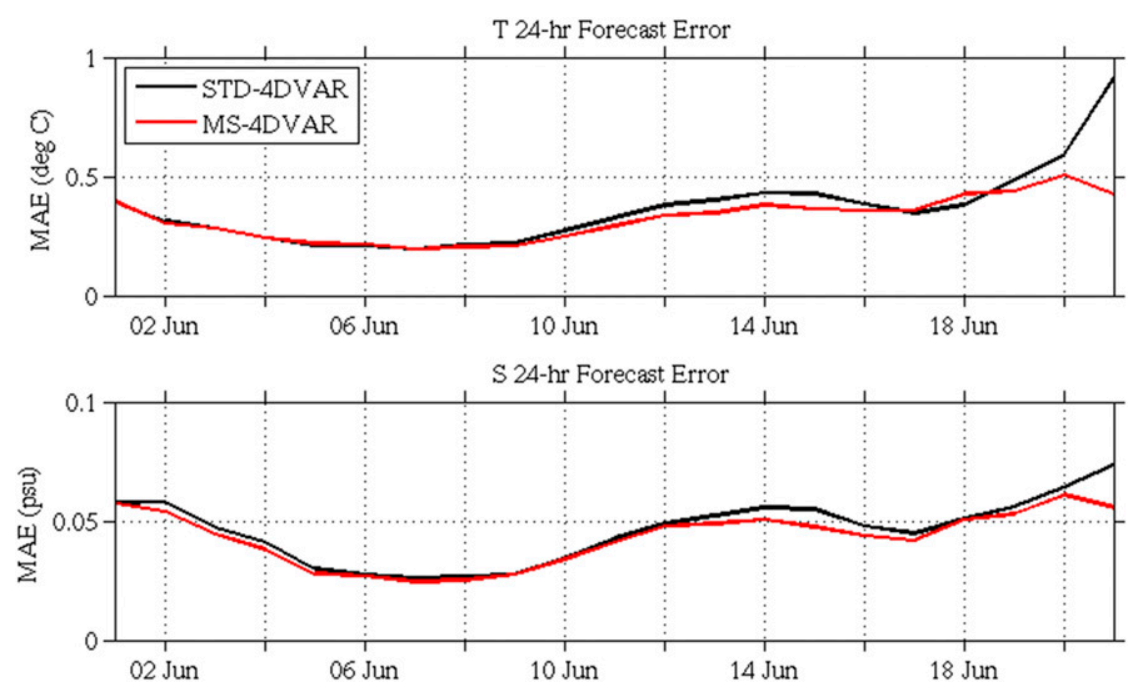

FIG. 13. The 24-h forecast MAE for STD-4DVAR (black lines) and MS-4DVAR (red lines) for (top) temperature and (bottom) salinity throughout the experiment time frame (1-21 Jun 2017). Values are computed by interpolating model 24 -h forecast fields to glider observation locations. Gliders from \pm 12 -h window around each forecast time are used in comparison. 

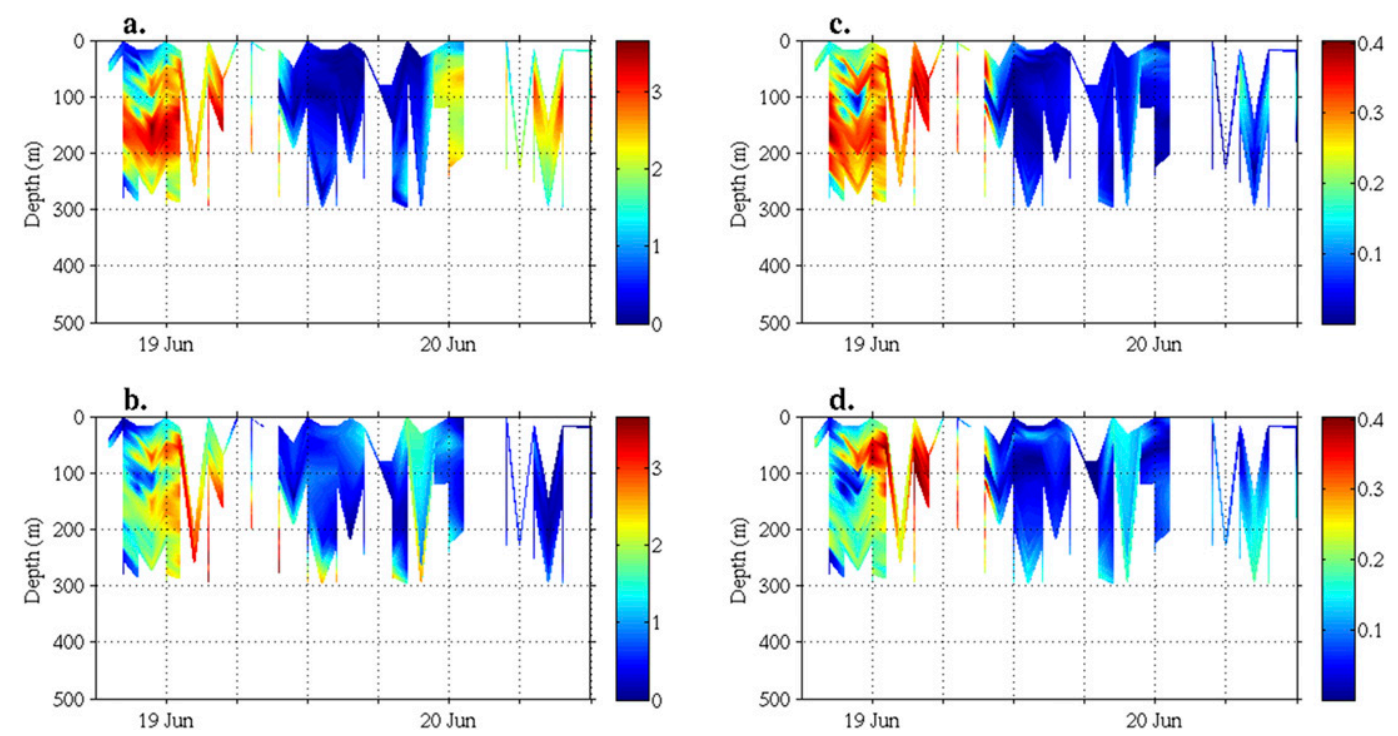

FIG. 14. Absolute difference between glider Maria observed and model forecast (a),(b) temperature and (c),(d) salinity from (top) the STD-4DVAR experiment and (bottom) the MS-4DVAR experiment from 19 to 21 Jun 2017. A total of 35 glider Maria profiles are shown.

(top panel) and salinity (bottom panel). As indicated by the analysis error results, the forecast prior to 10 June does not show much difference between the two experiments; likely because of the nature of the observations and the regions that are sampled. After 10 June, however, the 24-h forecast generated from the MS-4DVAR analyses begins to show lower error than that generated from STD-4DVAR in both temperature and salinity. This difference becomes enhanced by the end of the experiment, when the temperature forecast error from the STD-4DVAR analysis approaches $1.0^{\circ} \mathrm{C}$, while the forecast error from MS-4DVAR stays below $0.5^{\circ} \mathrm{C}$.

The lack of substantial differences in the forecast error can be partially explained by the nature of the observations used in the metric. Most of these observations are provided by the NREP-17 gliders, which are largely found either north or south of the IFF. Only briefly do the gliders cross the IFF (see Figs. 2-5). In most of these cases the forecast from the MS-4DVAR is similar to that of STD-4DVAR mainly because the IFF had not yet been sampled and assimilated by MS-4DVAR until after the gliders cross. There is one instance, however, where a glider from the NREP-17 dataset crosses the IFF twice: glider Maria. In this case, Maria transits the front around 12 June 2017 and again after 18 June 2017. It is after 18 June 2017 that the forecast MAE values shown from the MS-4DVAR experiment in Fig. 13 become discernably lower than that from STD-4DVAR. The forecast from each analysis method can be compared to the glider observations taken by Maria on these dates in the same manner as shown in Figs. 8 and 9.
A final analysis (Fig. 14), shows the absolute difference between the glider observed and closest-time model forecast temperature (left panels) and salinity (right panels) from the STD-4DVAR (top panels) and MS-4DVAR (bottom panels) experiments. The forecast fields from the STD-4DVAR analysis exhibit much higher difference with the Maria temperature and salinity observations than those from the forecast generated from the MS-4DVAR analysis. This suggests that the previous observations assimilated from gliders Maria, Noa, and Rose by the MS-4DVAR system helped to constrain the IFF in the vicinity of these gliders better than STD-4DVAR. A more definitive determination cannot be made, however, because of the lack of available independent observations. Overall, the forecast error time series indicates that the MS-4DVAR analyses do not degrade the forecasts relative to the forecast generated from STD-4DVAR. In fact, the forecasts from MS-4DVAR perform as well as those from STD-4DVAR and slightly better in regions that exhibit smaller-scale features and where there are observations to sample these features.

\section{Discussion and future work}

As ocean models are run at finer resolution it becomes necessary to constrain features at smaller scales. Ocean observing platforms, such as underwater gliders and the soon-to-be launched Surface Water and Ocean Topography (SWOT) wide-swath altimeter, are now (or will be) capable of collecting measurements that resolve 
these fine scales. Operational data assimilation algorithms, on the other hand, are still tuned for mesoscale features or larger. Li et al. (2015a,b) have done extensive work on deriving a $3 \mathrm{DVAR}$ system that can constrain small-scale features while still preserving the large-scale increment. The work shown here has extended this ability to the 4DVAR. Though the 4DVAR is able to resolve fine structures via the dynamic error covariance produced by the action of the tangent linear and adjoint model, it is still hindered by the use of a static covariance with a fixed correlation length scale. The multiscale 4DVAR approach, presented in this work, uses a multistep assimilation methodology to constrain small-scale features while also maintaining the proper analysis increment to the large scale. This is done by assimilating an averaged observation dataset with large static correlation length scales in the first assimilation step; then, using this analysis as the background for the second assimilation step, the MS-4DVAR assimilates the nonaveraged observation dataset with shorter static correlation length scales. The resulting analysis has been demonstrated here to not only fit the observations as well as the STD-4DVAR, but can also represent small-scale features in the observations that the STD-4DVAR method cannot. Further, there is a substantial increase in usable profile observations over STD-4DVAR when employing the MS-4DVAR algorithm (91\% of total profile observations for MS-4DVAR vs $12 \%$ for STD-4DVAR in this experiment). This increase in usable observations is significant when considering the cost in developing, maintaining, and deploying profile-collecting assets (such as gliders). Finally, the resulting 24-h forecast from the MS-4DVAR is as good or, at times, better than the forecast using an analysis from the STD-4DVAR. This is especially true for the region near the IFF as seen by the observations taken from glider Maria shown in Fig. 14.

The issue of observation overfitting has been mentioned at various points in this study, as well as the potential sources of overfitting (i.e., assimilation window overlap, lack of synoptic observations in the second assimilation step, and the lack of scale-based representation error in the two assimilation steps). It has been discussed how MS-4DVAR attempts to account for these potential problems, but a more thorough investigation (using a twin-assimilation experiment setup) will be conducted in future work.

A future paper is in preparation to use the MS-4DVAR system to assimilate glider observations from a unique network design. To more readily capture small-scale effects (e.g., internal waves) it has been proposed to operate gliders in small coordinated teams. This deployment strategy is well suited for the MS-4DVAR because of the proximity of glider observations in both time and space. NRL conducted such a field experiment off the North Carolina coast in May of 2017. This future study aims to evaluate the performance of MS-4DVAR using this new deployment strategy.

Acknowledgments. The authors thank the three anonymous reviewers for their comments, suggestions, and insight. Their constructive criticism improved the presentation and interpretation of the results shown in this article. This work was sponsored by the Office of Naval Research Program Element 73-6A04-A7-5 as part of the "Smart Glider Teams for Rapid Update of Local Analysis." This work was made possible, in part, by the NREP-17 sea trial including as participants CMRE, BODC, BOM, DGA, DSTL, FFI, IIM, METNO, MSS, NERSC, NOC, NRL, PML, RN, RSA, SAMS, Seiche, SHOM, UEA, UKHO, and UKMO. This paper is NRL paper Contribution Number NRL/JA/7320-17-3645.

\section{REFERENCES}

Bennett, A. F., 1992: Inverse Methods in Physical Oceanography. Cambridge University Press, 347 pp.

_ 2002: Inverse Modeling of the Ocean and Atmosphere. Cambridge University Press, $234 \mathrm{pp}$.

Capet, X., J. C. McWilliams, M. J. Molemaker, and A. F. Shchepetkin, 2008: Mesoscale to submesoscale transition in the California Current system. Part II: Frontal processes. J. Phys. Oceanogr., 38, 44-64, https://doi.org/10.1175/ 2007JPO3672.1.

Carrier, M. J., and H. Ngodock, 2010: Background-error correlation model based on the implicit solution of a diffusion equation. Ocean Modell., 35, 45-53, https://doi.org/10.1016/ j.ocemod.2010.06.003.

— - - S. Smith, G. Jacobs, P. Muscarella, T. Ozgokmen, B. Haus, and B. Lipphardt, 2014: Impact of assimilating ocean velocity observations inferred from Lagrangian drifter data using the NCOM-4DVAR. Mon. Wea. Rev., 142, 1509-1524, https://doi.org/10.1175/MWR-D-13-00236.1.

Chua, B. S., and A. F. Bennett, 2001: An inverse ocean modeling system. Ocean Modell., 3, 137-165, https://doi.org/10.1016/ S1463-5003(01)00006-3.

Courtier, P., 1997: Dual formulation of four-dimensional variational assimilation. Quart. J. Roy. Meteor. Soc., 123, 24492461, https://doi.org/10.1002/qj.49712354414.

Cummings, J. A., 2005: Operational multivariate ocean data assimilation. Quart. J. Roy. Meteor. Soc., 131, 3583-3604, https:// doi.org/10.1256/qj.05.105.

Fu, L.-L., and C. Ubelmann, 2014: On the transition from profile altimeter to swath altimeter for observing global ocean surface topography. J. Atmos. Oceanic Technol., 31, 560-568, https:// doi.org/10.1175/JTECH-D-13-00109.1.

Hogan, T. F., and Coauthors, 2014: The Navy Global Environmental Model. Oceanography, 27, 116-125, https://doi.org/ 10.5670/oceanog.2014.73.

Kantha, L. H., and C. A. Clayson, 2004: On the effect of surface gravity waves on mixing in an oceanic mixed layer 
model. Ocean Modell., 6, 101-124, https://doi.org/10.1016/ S1463-5003(02)00062-8.

Li, Z., J. C. McWilliams, K. Ide, and J. D. Farrar, 2015a: Coastal ocean data assimilation using a multi-scale three-dimensional variational scheme. Ocean Dyn., 65, 1001-1015, https:// doi.org/10.1007/s10236-015-0850-x.

_,$-\ldots$, and J. D. Farrara, 2015b: A multiscale variational data assimilation scheme: Formulation and illustration. Mon. Wea. Rev., 143, 3804-3822, https://doi.org/10.1175/ MWR-D-14-00384.1.

Marchesiello, P., J. C. McWilliams, and A. F. Shchepetkin, 2001: Open boundary conditions for long-term integration of regional oceanic models. Ocean Modell., 3, 1-20, https://doi.org/ 10.1016/S1463-5003(00)00013-5.

Martin, P. J., 2000: Description of the Navy Coastal Ocean Model version 1.0. NRL Rep. NRL/FR/7322/00/9962, 45 pp. [Available from NRL, Code 7322, Bldg. 1009, Stennis Space Center, MS 39529-5004.]

Miyazawa, Y., and Coauthors, 2017: Assimilation of highresolution sea surface temperature data into an operational nowcast/forecast system around Japan using a multi-scale three-dimensional variational scheme. Ocean Dyn., 67, 713-728, https://doi.org/10.1007/s10236-0171056-1.

Muscarella, P. A., M. J. Carrier, and H. E. Ngodock, 2014: An examination of a multi-scale three-dimensional variational data assimilation scheme in the Kuroshio Extension using the Naval Coastal Ocean Model. Cont. Shelf Res., 73, 41-48, https://doi.org/10.1016/j.csr.2013.11.009.

Ngodock, H. E., and M. J. Carrier, 2013: A weak constraint 4D-Var Assimilation system for the Navy coastal ocean model using the representer method. Data Assimilation for Atmospheric, Oceanic and Hydrologic Applications, Vol. II, S. K. Park and L. Xu, Eds., Springer-Verlag, 367-390, https://doi.org/10.1007/ 978-3-642-35088-7_15.
, and - 2014: A 4DVAR system for the Navy Coastal Ocean Model. Part I: System description and assimilation of synthetic observations in Monterey Bay. Mon. Wea. Rev., 142, 2085-2107, https://doi.org/10.1175/MWR-D-13-00221.1.

Paduan, J. D., and H. C. Graber, 1997: Introduction to highfrequency radar: Reality and myth. Oceanography, 10, 36-39, https://doi.org/10.5670/oceanog.1997.18.

Rudnick, D. L., R. E. Davis, C. C. Eriksen, D. M. Fratantoni, and M. J. Perry, 2004: Underwater gliders for ocean research. Mar. Technol. Soc. J., 38, 73-84, https://doi.org/10.4031/ 002533204787522703.

Shchepetkin, A. F., and J. C. McWilliams, 2003: A method for computing horizontal pressure-gradient force in an oceanic model with nonaligned vertical coordinate. J. Geophys. Res., 108, 3090, https://doi.org/10.1029/2001JC001047.

- and - 2005: The regional oceanic modeling system (ROMS): A split-explicit, free-surface, topography-followingcoordinate oceanic model. Ocean Modell., 9, 347-404, https:// doi.org/10.1016/j.ocemod.2004.08.002.

Smagorinsky, J., 1963: General circulation experiments with the primitive equations. I: The basic experiment. Mon. Wea. Rev., 91, 99-164, https://doi.org/10.1175/1520-0493(1963)091<0099: GCEWTP $>2.3 . \mathrm{CO} ; 2$.

Weaver, A. T., and P. Courtier, 2001: Correlation modeling on the sphere using a generalized diffusion equation. Quart. J. Roy. Meteor. Soc., 127, 1815-1846, https://doi.org/10.1002/qj.49712757518.

Xie, Y., S. Koch, J. McGinley, S. Albers, P. E. Bieringer, M. Wolfson, and M. Chan, 2011: A space-time multiscale analysis system: A sequential variational analysis approach. Mon. Wea. Rev., 139, 1224-1240, https://doi.org/10.1175/ 2010MWR3338.1.

Xu, Q., L. Wei, J. Gao, Q. Zhao, K. Nai, and S. Liu, 2016: Multistep variational data assimilation: Important issues and a spectral approach. Tellus, 68A, 31110, https://doi.org/10.3402/ tellusa.v68.31110. 